\title{
Intracoronary infusion of Wharton's jelly-derived crossanat mesenchymal stem cells in acute myocardial infarction: double-blind, randomized controlled trial
}

Lian R Gao ${ }^{1 *}$, Yu Chen ${ }^{1}$, Ning K Zhang ${ }^{1}$, Xi L Yang ${ }^{2}$, Hui L Liư ${ }^{3}$, Zhi G Wang ${ }^{1}$, Xiao Y Yann ${ }^{5}$, Yu Wang ${ }^{7}$, Zhi M Zhu', Tian C Li ${ }^{1}$, Li H Wang ${ }^{1}$, Hai Y Chen ${ }^{1}$, Yun D Chen ${ }^{6}$, Chao L Huang ${ }^{8}$, Peng Qu' ${ }^{9}$ Chen Yao ${ }^{5}$, Bin Wang ${ }^{10}$,

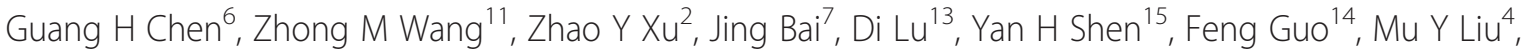
Yong Yang ${ }^{3}$, Yan C Ding ${ }^{9}$, Ye Yang ${ }^{1}$, Hai T Tian', Qing A Ding ${ }^{1}$, Li N Li ${ }^{8}$, Xin C Yang ${ }^{12}$ and Xiang $\mathrm{Hu}^{4^{*}}$

\begin{abstract}
Background: The use of adult stem cells is limited by the quality and quantity of host stem cells. It has been demonstrated that Wharton's jelly-derived mesenchymal stem cells (WJMSCs), a primitive stromal population, could integrate into ischemic cardiac tissues and significantly improve heart function. In this randomized, controlled trial, our aim was to assess the safety and efficacy of intracoronary WJMSCs in patients with ST-elevation acute myocardial infarction (AMI).

Methods: In a multicenter trial, 116 patients with acute ST-elevation MI were randomly assigned to receive an intracoronary infusion of WJMSCs or placebo into the infarct artery at five to seven days after successful reperfusion therapy. The primary endpoint of safety: the incidence of adverse events (AEs) within 18 months, was monitored and quantified. The endpoint of efficacy: the absolute changes in myocardial viability and perfusion of the infarcted region from baseline to four months, global left ventricular ejection fraction (LVEF) from baseline to 18 months were measured using F-18-fluorodeoxyglucose positron emission computed tomography (F-18-FDG-PET) and 99mTc-sestamibi single-photon emission computed tomography (99mTc-SPECT), and two-dimensional echocardiography, respectively.
\end{abstract}

Results: During 18 months follow-up, AEs rates and laboratory tests including tumor, immune, and hematologic indexes were not different between the two groups. The absolute increase in the myocardial viability (PET) and perfusion within the infarcted territory (SPECT) was significantly greater in the WJMSC group $[6.9 \pm 0.6 \%(95 \% \mathrm{Cl}$, 5.7 to 8.2$)$ ] and [7.1 $\pm 0.8 \%(95 \% \mathrm{Cl}, 5.4$ to 8.8$)$ than in the placebo group [3.3 $\pm 0.7 \%(95 \% \mathrm{Cl}, 1.8$ to 4.7$), \mathrm{P}<0.0001]$ and $3.9 \pm 0.6(95 \% \mathrm{Cl}, 2.8$ to 5.0), $\mathrm{P}=0.002]$ at four months. The absolute increase in the LVEF at 18 months in the WJMSC group was significantly greater than that in the placebo group [7.8 \pm 0.9 (6.0 to approximately 9.7) vs. $2.8 \pm 1.2$ ( 0.4 to approximately 5.1), $\mathrm{P}=0.001$ ]. Concomitantly, the absolute decreases in LV end-systolic volumes and end-diastolic volumes at 18 months in the WJMSC group were significantly greater than those in the placebo group $(P=0.0004, P=0.004$, respectively).

(Continued on next page)

\footnotetext{
* Correspondence: lianrugao668@yahoo.com; huxiang@beike.cc

${ }^{1}$ Center of Cardiology, Navy General Hospital, Beijing, China

${ }^{4}$ Shenzhen Beike Cell Engineering Research Institute, Guangdong Province, Shenzhen, China

Full list of author information is available at the end of the article
}

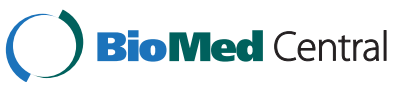

(c) 2015 Gao et al. This is an Open Access article distributed under the terms of the Creative Commons Attribution License (http://creativecommons.org/licenses/by/4.0), which permits unrestricted use, distribution, and reproduction in any medium, provided the original work is properly credited. The Creative Commons Public Domain Dedication waiver (http:// creativecommons.org/publicdomain/zero/1.0/) applies to the data made available in this article, unless otherwise stated. 
(Continued from previous page)

Conclusions: Intracoronary infusion of WJMSCs is safe and effective in patients with AMI, providing clinically relevant therapy within a favorable time window. This study encourages additional clinical trials to determine whether WJMSCs may serve as a novel alternative to BMSCs for cardiac stem cell-based therapy.

Trial registration: Clinical Trials NCT01291329 (02/05/2011).

Keywords: Myocardial infarction, Mesenchymal stem cells, Wharton's jelly of umbilical cord

\section{Background}

Coronary artery disease (CAD) remains a major worldwide cause of morbidity and mortality [1]. Despite recent advances in treatments for acute myocardial infarction (AMI), the irreversible loss of cardiomyocytes after an AMI leads to left ventricular (LV) remodeling and ischemic heart failure [1]. Regenerative cell therapies are emerging as potential treatments for AMI [2]. Studies have demonstrated the ability of transplanted mesenchymal stem cells (MSCs) to engraft, differentiate into cardiomyocyte-like and endothelial cells, and recruit endogenous cardiac stem cells [3-6]. Clinical evidence has shown that the intracoronary delivery of bone marrow mononuclear cells (BMMCs) or bone marrow mesenchymal stem cells (BMSCs) can improve the ejection fraction and reduce the infarct size [7-11]. However, the viability and function of autologous adult stem cells decline with age, especially in patients with MI [12, 13], which significantly limits their viability for clinical transplantation during the optimal window of opportunity to prevent adverse left ventricular remodeling [12]. Therefore, alternative sources of stem cells must be explored.

Wharton's jelly-derived mesenchymal stem cells (WJMSCs), a primitive stromal population [14, 15], have been isolated from a continuum from the sub-amnion to the perivascular region of the umbilical cord $[14,15]$. Wharton's jelly of the umbilical cord originates from the extraembryonic and/or the embryonic mesoderm at day 13 of embryonic development [15]. WJMSCs retain a combination of most of their embryonic stem cell (ESC) and MSC markers in primary culture and early passages, thus retaining their multipotent stem cell characteristics [15-17]. Using Affymetrix GeneChip microarray and functional network analyses, we found for the first time that WJMSCs, except for their expression of stemness molecular markers in common with human ESCs (hESCs), exhibited a high expression of early cardiac transcription factor genes and could be induced to differentiate into cells expressing cardiac a-actin, troponin $\mathrm{T}$ and connexin43 in vitro [18]. Moreover, growing evidence has shown that WJMSCs can be induced to differentiate into cardiomyocytes and endothelial cells and to integrate into the vasculature and ischemic cardiac tissue, as well as to improve heart function significantly [19-22].
In contrast to autologous adult stem cells, WJMSCs display greater cardiovascular differentiation potential [18-23], and more importantly, they are immune-privileged and can be transplanted into unrelated recipients [24]. This suggests the possibility of an allogeneic, "off-the-shelf" cell product, which can be used during the optimal time-frame for stem cell-based therapies after AMI, or even applied directly following revascularization of the AMI [15-17, 24]. WJMSCs constitute an attractive alternative to autologous MSCs for stem cell-based cardiac therapies [25].

Although our pilot clinical trial [26] and other clinical studies [10] using allogeneic stem cells showed promising results, there is no convincing evidence to date of the therapeutic safety and efficacy of the use of WJMSCs in humans. For this reason, this randomized, double-blind, multicenter trial was performed to investigate the therapeutic safety and efficacy of WJMSCs in patients with STelevation AMI.

\section{Methods}

\section{Study population}

Patients with ST-elevation AMI were admitted to cardiology centers in 11 hospitals in China between February 2011 and January 2012. A total of 160 subjects were enrolled in the trial. For inclusion in the study, patients had to satisfy the following eligibility criteria: 18 - to 80 -years old; a first STsegment elevation MI; successful reperfusion with stent implantation of the infarct-related artery within 12 hours after the onset of symptoms; a substantial residual LV regional wall-motion abnormality (three or more hypokinetic LV segments observed on an echocardiograph after percutaneous coronary intervention, PCI); and creatine kinase (CK)$\mathrm{MB}$ levels over three-fold the upper limit of the reference values. Exclusion criteria included previous Q-wave MI and severe coexisting conditions, such as advanced renal or hepatic dysfunction, and documented terminal illness or cancer. All subjects were administered medications according to the current updated ACC/AHA/SCAI guidelines along with standard rehabilitation programs for MI [27].

The study protocol conformed to the Declaration of Helsinki and was approved by the ethics committee of Navy General Hospital. All subjects signed written informed consent for enrollment in the study and treatment. The trial was monitored by an independent data and safety 
monitoring board (DSMB) who met every two months and as needed to assess adverse events.

\section{Study design and treatment randomization}

The eligible patients were assigned randomly to each of two groups (WJMSCs or placebo control) in a 1:1 fashion using a computer-generated randomization of sequence numbers (Fig. 1). Physicians and other clinical personnel remained blind to the treatment assignment throughout the study. Day 0 was defined as the day of PCI. During days 5-7, all subjects were assigned randomly into either the WJMSC group, receiving $6 \times 10^{6}$ WJMSCs through intracoronary infusion as described previously [28], or the placebo group, with a placebo injected via the same delivery method as that of the WJMSC group. Safety was evaluated on days 0 and 3, as well as 1, 4, 12 and 18 months post-treatment. Cardiac nuclear studies were performed pre-treatment and at four months post-treatment. Twodimensional echocardiograms were measured before cell transfer and 4, 12 and 18 months after cell transplantation or placebo infusion.

\section{Study end points}

The primary end point was safety: the incidence of adverse events (AEs) within 18 months, including death, nonfatal MI, stroke, hospitalization for worsening heart function, severe arrhythmias, repeated coronary intervention, stent thrombosis, coronary artery microvascular obstruction, immune system disorders, or ectopic tissue formation, was monitored and quantified. Laboratory assays, including biochemical assays, hematologic, tumor and immune indexes and Holter monitoring, were performed at the different follow-up times specified above.

The secondary end point was efficacy, which was assessed in terms of the absolute change in myocardial viability and perfusion in the infarcted region from the baseline to 4 months post-treatment, as well as the global LV ejection fraction (LVEF) from baseline to 18 months post-treatment, as measured by F-18-fluorodeoxyglucose positron emission computed tomography (F-18-FDGPET), 99mTc-sestamibi single-photon emission computed tomography (99mTc-SPECT) and two-dimensional echocardiogram (ECG), respectively.

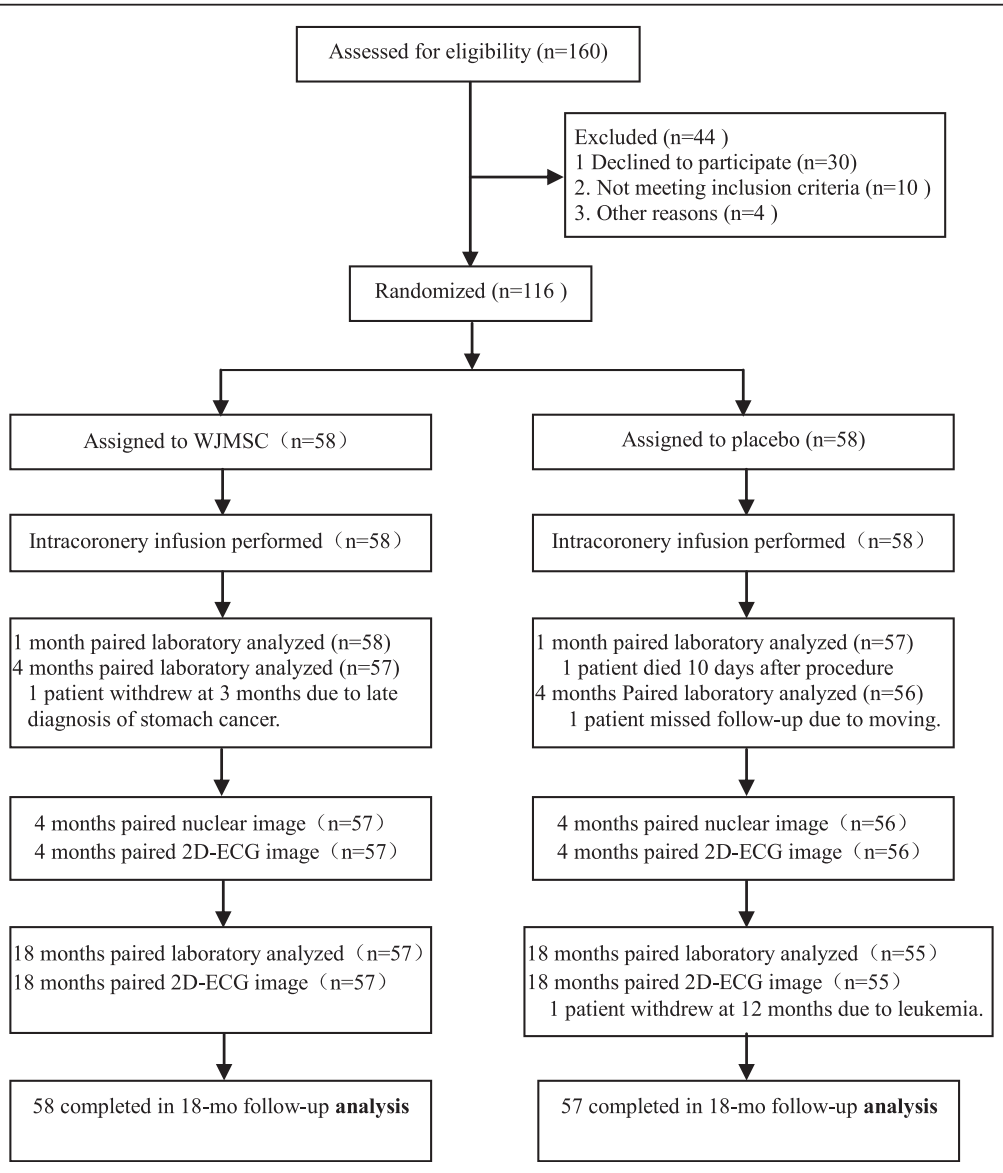

Fig. 1 Enrollment and outcomes 
Table 1 Baseline characteristics of the patients

\begin{tabular}{l} 
Characteristic \\
\hline Risk factor \\
Age (years) \\
Men - number (\%) \\
Body-mass index (kg/m²) \\
Diabetes mellitus - number (\%) \\
Hyperlipidemia - number (\%) \\
Hypertension - number (\%) \\
Smoking (current of former) -number (\%) \\
Family history of coronary heart disease - number (\%)
\end{tabular}

Placebo
$(n=58)$

$56.7+$

Coronary artery disease - number (\%)

$\begin{array}{lll}56.7 \pm 1.7 & 57.3 \pm 1.3 & 0.79 \\ 51(87.9) & 55(94.8) & 0.18 \\ 25.4 \pm 0.3 & 24.9 \pm 0.3 & 0.26 \\ 14(24.1) & 17(29.3) & 0.52 \\ 22(37.9) & 21(36.2) & 0.84 \\ 26(44.8) & 33(56.9) & 0.19 \\ 32(55.2) & 34(58.6) & 0.70 \\ 20(34.5) & 17(29.3) & 0.55 \\ & & 0.19\end{array}$

\footnotetext{
1 -Vessel disease

2-Vessel disease

3-Vessel disease
}

28(48.3)

21(36.2)

16(27.6)

$14(24.1)$

14(24.1)

23(39.7)

\section{Infarct treatment}

Infarct-related artery - number (\%)

Left anterior descending artery

31(53.4)

29(50.0)

Left circumflex artery

6(10.3)

10(17.2)

Right coronary artery

21(36.2)

19(32.8)

PCI for additional stenoses in non-infarct-related vessels - number (\%)

$10(17.2)$

15(25.9)

0.25

Time from symptom onset to first reperfusion therapy-hour

Mean

Median

6.3

Drug-eluting stent - number (\%)

Gp II b/llla inhibitor during acute PCI - number (\%)

Intravenous catecholamines - number (\%)

3(5.2)

$8(13.8)$

0.78

Maximal creatine kinase (U/L)

Maximal creatine kinase $M B(U / L)$

$192 \pm 21$

$1653 \pm 206$

0.93

Maximal troponin $\mathrm{T}(\mu \mathrm{g} / \mathrm{L})$

$18.2 \pm 3.1$

$170 \pm 17$

0.42

TIMI flow grade

$24.7 \pm 4.1$

Before PCI - number (\%)

Grade 0 or 1

42(72.4)

$42(72.4)$

Grade 2

$8(13.8)$

$7(12.1)$

Grade 3

$9(15.5)$

After $\mathrm{PCl}$ - number (\%)

Grade 0 or 1

$0(0)$

Grade 2

$1(1.7)$

$0(0)$

Grade 3

57(98.3)

\section{Baseline quantitative measure of LV function}

Global left ventricular ejection fraction (\%)

Mean

Left venticular fractional shortening (\%)

$$
\text { Mean }
$$


Table 1 Baseline characteristics of the patients (Continued)

\begin{tabular}{|c|c|c|c|}
\hline Mean & $1.28 \pm 0.03$ & $1.29 \pm 0.02$ & 0.62 \\
\hline \multicolumn{4}{|l|}{ End-systolic volume (ml) } \\
\hline Mean & $64.6 \pm 2.8$ & $63.3 \pm 2.7$ & 0.68 \\
\hline \multicolumn{4}{|l|}{ End-diastolic volume (ml) } \\
\hline Mean & $129.9 \pm 3.5$ & $130.4 \pm 3.6$ & 0.90 \\
\hline \multicolumn{4}{|l|}{ Cell therapy } \\
\hline \multicolumn{4}{|c|}{ Time from reperfusion to infusion of study therapy-days } \\
\hline Mean & $6.3 \pm 0.1$ & $6.1 \pm 0.1$ & 0.25 \\
\hline Median & 6.5 & 6.0 & \\
\hline \multicolumn{4}{|l|}{ TIMI flow grade before study therapy } \\
\hline Mean & $2.98 \pm 0.02$ & $2.97 \pm 0.02$ & 0.56 \\
\hline Median & 3 & 3 & \\
\hline \multicolumn{4}{|l|}{ TIMI flow grade after study therapy } \\
\hline Mean & $2.98 \pm 0.02$ & $2.97 \pm 0.02$ & 0.56 \\
\hline Median & 3 & 3 & \\
\hline \multicolumn{4}{|l|}{ Current medication - number (\%) } \\
\hline Aspirin and clopidogrel & $58(100)$ & $58(100)$ & 1 \\
\hline Beta-blocker & 48(82.8) & $42(72.4)$ & 0.18 \\
\hline ACE-inhibitors or angiotensin-receptor B & $43(74.1)$ & $42(72.4)$ & 0.83 \\
\hline Stains & $54(93.1)$ & 54(93.1) & 1 \\
\hline
\end{tabular}

\section{Preparation and administration of WJMSCs}

The protocol of WJMSCs preparation was approved by the General Logistics Department of the PLA and the Navy General Hospital Ethical Review Board. Twenty-one human umbilical cords were obtained, with the consent of the parents, from healthy donors, who had no complications throughout the pregnancy, no history of disease, and a full-term birth by caesarian section, and were aseptically stored in sterile saline and processed within six hours from partum to obtain the umbilical cord. After removal of blood vessels, the abundant extracellular matrix of Wharton's jelly, which is a mucous tissue continuum from the subamnion to the perivascular region, was scraped off with a scalpel, finely cut and rinsed in sterile phosphatebuffered saline. The WJMSCs were isolated by a nonenzymatic method and cultured as described previously [18]. The WJMSCs were purified in a central cellprocessing laboratory following the regulatory guidelines of the International Conference on Harmonization and the US Food and Drug Administration [29]. All procedures were performed and all solutions were prepared under Good Manufacturing Practice (GMP).

The infused WJMSCs were harvested at passage 3, during which $\geq 95 \%$ of cells expressed CD29, CD73, CD90 and CD105, while the expression of CD45, CD34, CD14, CD79 and HLA-DR was $2 \%$ or less. Released cells were negative for the pathogenic microorganisms $\mathrm{HBV}, \mathrm{HCV}, \mathrm{HIV}$, cytomegalovirus, syphilis and exhibited ALT and endotoxin levels within $40 \mathrm{IU} / \mathrm{L}$ and $0.5 \mathrm{EU} /$ $\mathrm{mL}$, respectively. Final processing incorporated a total cell count and cell viability $(\geq 85 \%)$ determination by trypan blue testing. Based on the results from our animal experiments, we decided on a dose of $6 \times 10^{6}$ WJMSCs by intracoronary transplantation in this trial. In brief, a dose-escalation study for intracoronary delivery of WJMSCs involved 12 pig models of AMI, weighing $28-35 \mathrm{~kg}$, of mixed gender. The dose was escalated at $1,2,3,6 \times 10^{6}$ with a 30-minute interval. A coronary angiogram as well as left ventriculogram was obtained at 15 minutes following each infusion. Blood flow to the distal left anterior descending artery (LAD), measured under fluoroscopy by counting the number of heart beats required to fill this region of the vessel with contrast, was not changed at bolus doses up to $6 \times 10^{6}$ WJMSCs. Significant changes in LV wall motion were revealed until administration of the $3 \times 10^{6}$ dose [30]. The placebo consisted of a vehicle (saline with 10,000 U/L heparin) injected without cells.

The cells were shipped at $4{ }^{\circ} \mathrm{C}$ and delivered to each catheterization laboratory at the 11 participating cardiology centers, using a standard operating procedure. After extensive discussion with the enrolled subjects, 
Table 2 Clinical events during the 18 months follow-up period

\begin{tabular}{|c|c|c|c|}
\hline Event & Placebo & WJMSCS & $P$ value \\
\hline Events during procedure & $(n=58)$ & $(n=58)$ & \\
\hline Death & 0 & 0 & \\
\hline Obstruction of related-vessel & 0 & 0 & \\
\hline Severe arrhythmia & 0 & 0 & \\
\hline Events 6-mo follow-up (cumulative) ${ }^{a}$ & $(n=57)$ & $(\mathrm{n}=58)$ & \\
\hline Death & 1 & 0 & $0.49^{\mathrm{b}}$ \\
\hline Recurrence Ml & 0 & 0 & \\
\hline Rehospitalization for heart failure & 0 & 1 & $1.0^{\mathrm{b}}$ \\
\hline Stent thrombosis & 0 & 0 & \\
\hline Revascularization & 0 & 1 & $1.0^{\mathrm{b}}$ \\
\hline Cerebral infarction & 0 & 0 & \\
\hline Arrhythmia & 0 & 0 & \\
\hline \multicolumn{4}{|l|}{ Immune system disorder } \\
\hline Ectopic tissue formation & 0 & 1 & $1.0^{\mathrm{b}}$ \\
\hline \multicolumn{4}{|l|}{ Combined events } \\
\hline Death or Ml & 1 & 0 & $0.49^{\mathrm{b}}$ \\
\hline Death, recurrence of $\mathrm{Ml}$, revascularization procedure & 1 & 1 & $1^{\mathrm{b}}$ \\
\hline Death, Ml, or rehospitalization for heart failure & 1 & 1 & $1^{\mathrm{b}}$ \\
\hline Death, MI, stroke, rehospitalization for heart failure, severe arrhythmia & 1 & 1 & $1^{\mathrm{b}}$ \\
\hline Immune system disorder, ectopic tissue formation & 0 & 1 & $1^{\mathrm{b}}$ \\
\hline \multicolumn{4}{|l|}{ Events 12-mo follow-up (cumulative) } \\
\hline Death & 1 & 0 & $0.49^{\mathrm{b}}$ \\
\hline Recurrence Ml & 0 & 0 & \\
\hline Rehospitalization for heart failure & 0 & 1 & $1.0^{\mathrm{b}}$ \\
\hline Stent thrombosis & 0 & 0 & \\
\hline Revascularization & 0 & 1 & $1.0^{\mathrm{b}}$ \\
\hline Cerebral infarction & 0 & 0 & \\
\hline Arrhythmia & 0 & 0 & \\
\hline Immune system disorder & 0 & 0 & \\
\hline Ectopic tissue formation & 1 & 1 & $1.0^{\mathrm{b}}$ \\
\hline \multicolumn{4}{|l|}{ Combined events } \\
\hline Death or Ml & 1 & 0 & $0.49^{\mathrm{b}}$ \\
\hline Death, recurrence of $\mathrm{Ml}$, revascularization procedure & 1 & 1 & $1^{\mathrm{b}}$ \\
\hline Death, MI, or rehospitalization for heart failure & 1 & 1 & $1^{\mathrm{b}}$ \\
\hline Death, MI, stroke, rehospitalization for heart failure, severe arrhythmia & 1 & 1 & $1^{\mathrm{b}}$ \\
\hline Immune system disorder, ectopic tissue formation & 1 & 1 & $1^{\mathrm{b}}$ \\
\hline \multicolumn{4}{|l|}{ Events 18-mo follow-up (cumulative) } \\
\hline Death & 1 & 0 & $0.49^{\mathrm{b}}$ \\
\hline Recurrence Ml & 0 & 0 & \\
\hline Rehospitalization for heart failure & 0 & 1 & $1.0^{\mathrm{b}}$ \\
\hline Stent thrombosis & 0 & 0 & \\
\hline Revascularization & 0 & 1 & $1.0^{\mathrm{b}}$ \\
\hline Cerebral infarction & 0 & 0 & \\
\hline Arrhythmia & 0 & 0 & \\
\hline
\end{tabular}


Table 2 Clinical events during the 18 months follow-up period (Continued)

\begin{tabular}{|c|c|c|c|}
\hline Immune system disorder & 0 & 0 & \\
\hline Ectopic tissue formation & 1 & 1 & $1.0^{\mathrm{b}}$ \\
\hline \multicolumn{4}{|l|}{ Combined events } \\
\hline Death or Ml & 1 & 0 & $0.49^{b}$ \\
\hline Death, recurrence of $\mathrm{Ml}$, revascularization procedure & 1 & 1 & $1^{\mathrm{b}}$ \\
\hline Death, MI, or rehospitalization for heart failure & 1 & 1 & $1^{\mathrm{b}}$ \\
\hline Death, MI, stroke, rehospitalization for heart failure, severe arrhythmia & 1 & 1 & $1^{\mathrm{b}}$ \\
\hline Immune system disorder, ectopic tissue formation & 1 & 1 & $1^{\mathrm{b}}$ \\
\hline
\end{tabular}

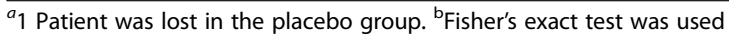

MI myocardial infarction

written informed consent was obtained before initiating treatment. After arterial puncture and administration of $8000 \mathrm{U}$ heparin, $6 \times 10^{6}$ WJMSCs dispersed in $10 \mathrm{~mL}$ heparinized saline (saline with 10,000 U/L heparin), or the placebo, were infused using a stop-flow technique through an over-the-wire balloon catheter positioned within the stent segment as described previously [28]. Contrast medium was injected into the infarct-related artery to ascertain vessel patency after cell infusion.

\section{F-18-FDG-PET and 99mTc-sestamibi SPECT examination}

F-18-FDG- PET (GE Millennum VG Hawkeye, Israel) and 99mTc-sestamibi SPECT (Varicam, GE-Elscint, Haifa, Israel) was performed in patients at one day before and four months after the procedure. After an overnight fast for at least 12 hours, patients were given an oral glucose load according to their serum glucose level. Sequential measurements of serum glucose were made until the serum glucose level reached $7.8-8.9 \mathrm{mmol} / \mathrm{L}$ and then 18F-FDG (222-296 MBq) and 99mTc-sestamibi (555-740 $\mathrm{MBq}$ ) were injected intravenously, respectively. Myocardial images were reconstructed using a standard filtered back projection and displayed as a series of short-axis, horizontal and vertical long-axis slices. Mean signal intensities were measured in the respective areas supplied by the three major coronary arteries in three-axis views. Results were calculated using F-18-FDG-PET and 99mTcsestamibi SPECT bull's-eye views. All parameters were analyzed independently by two experienced observers who were unaware of the treatment assignment.

\section{Two-dimensional echocardiogram examination}

Patients who received cell grafts or standard treatment underwent echocardiography consecutively $1-2$ days before cell/placebo infusion and at 4,12 and 18 months follow-up. A 17-segment echocardiogram was performed to measure regional left ventricular wall motion score, end-systolic volume, end-diastolic volume and LVEF by using standard methods of the American Society of Echocardiography [31], and were analyzed independently by two experienced observers who were unaware of patients' treatment assignments.

\section{Statistical analyses}

The population size of patient enrollment was influenced by the difficulty in selecting eligible patients because the inclusion criteria used in our study selected only those patients with AMI who had undergone primary PCI within 12 hours and who had also agreed to accept a WJMSC intracoronary transplant five days after their first PCI operation. Very few patients were deemed eligible. To address this issue, we expanded the study to include more hospitals. We also performed a statistical analysis specifically to determine whether the study population was normally distributed. To ascertain the efficacy, including PET and SPECT at month 4, and two dimensional-ECG at months 4, 12 and 18, with variables adjusted by baseline values, analysis of covariance (ANCOVA) was performed to assess differences between the placebo and WJMSC treatment groups. To estimate the treatment effect, differences in least-squares means and the corresponding $95 \%$ confidence intervals (CI) were calculated based on the ANCOVA model. The Wilcoxon or Student's t-test was used to compare changes between the baseline and follow-up values according to the distribution of variables. Categorical variables were analyzed by chi-square or Fisher's exact test, as appropriate. Continuous variables were expressed as means \pm standard errors (SE) unless otherwise stated. Categorical data were presented as frequencies and percentages. All statistical tests were two-sided, and $\mathrm{P}<0.05$ was considered statistically significant. All analyses were performed using SAS software, version 9.3 (SAS Institute, Cary, NC, USA).

\section{Results}

\section{Participant characteristics}

A total of 160 subjects with ST-elevation AMI were screened, and 116 eligible subjects signed written informed consent to participate in the study. All patients 
Table 3 Quantitative measures of blood index

\begin{tabular}{|c|c|c|c|}
\hline Blood index & $\begin{array}{l}\text { Placebo } \\
\text { (number = 55) }\end{array}$ & $\begin{array}{l}\text { WJMSCs } \\
\text { (number = 57) }\end{array}$ & $P$ value \\
\hline \multicolumn{4}{|l|}{$\mathrm{CD} 3(\%)$} \\
\hline Baseline & $66.8 \pm 0.9$ & $67.6 \pm 0.8$ & 0.48 \\
\hline 3 Days & $67.6 \pm 1.1$ & $69.1 \pm 0.9$ & 0.29 \\
\hline$P$ value(baseline vs.3d) & 0.43 & 0.08 & \\
\hline $1 \mathrm{Mo}$ & $67.3 \pm 1.0$ & $66.6 \pm 0.9$ & 0.53 \\
\hline P value (baseline vs.1 mo) & 0.58 & 0.18 & \\
\hline $4 \mathrm{Mo}$ & $65.2 \pm 0.7$ & $66.3 \pm 0.9$ & 0.34 \\
\hline P value (baseline vs. 6 mo) & 0.06 & 0.11 & \\
\hline $12 \mathrm{Mo}$ & $66.2 \pm 0.9$ & $66.4 \pm 0.8$ & 0.81 \\
\hline P value (baseline vs.12 mo) & 0.64 & 0.16 & \\
\hline $18 \mathrm{Mo}$ & $67.1 \pm 1.0$ & $66.8 \pm 0.9$ & 0.76 \\
\hline P value (baseline vs.18 mo) & 0.66 & 0.29 & \\
\hline \multicolumn{4}{|l|}{ CD4(\%) } \\
\hline Baseline & $39.6 \pm 1.2$ & $40.1 \pm 0.8$ & 0.70 \\
\hline 3 Days & $40.4 \pm 1.1$ & $39.3 \pm 0.8$ & 0.39 \\
\hline$P$ value (baseline vs.3 d) & 0.37 & 0.24 & \\
\hline $1 \mathrm{Mo}$ & $38.2 \pm 1.0$ & $37.1 \pm 0.7$ & 0.34 \\
\hline P value (baseline vs.1 mo) & 0.09 & $<0.0001$ & \\
\hline $4 \mathrm{Mo}$ & $37.7 \pm 1.1$ & $38.0 \pm 0.8$ & 0.75 \\
\hline P value (baseline vs.6 mo) & 0.09 & 0.02 & \\
\hline $12 \mathrm{Mo}$ & $37.6 \pm 0.9$ & $38.2 \pm 0.6$ & 0.58 \\
\hline$P$ value (baseline vs.12 mo) & 0.11 & 0.03 & \\
\hline $18 \mathrm{Mo}$ & $38.8 \pm 1.1$ & $39.2 \pm 0.9$ & 0.45 \\
\hline P value (baseline vs.18 mo) & 0.09 & 0.16 & \\
\hline \multicolumn{4}{|l|}{ CD8(\%) } \\
\hline Baseline & $21.9 \pm 0.9$ & $22.4 \pm 0.8$ & 0.39 \\
\hline 3 Days & $21.3 \pm 0.8$ & $22.9 \pm 0.8$ & 0.13 \\
\hline$P$ value (baseline vs.3 d) & 0.36 & 0.16 & \\
\hline $1 \mathrm{Mo}$ & $21.5 \pm 0.5$ & $22.8 \pm 0.7$ & 0.14 \\
\hline P value (baseline vs.1 mo) & 0.63 & 0.47 & \\
\hline $4 \mathrm{Mo}$ & $21.5 \pm 0.7$ & $21.8 \pm 0.7$ & 0.78 \\
\hline P value (baseline vs. 6 mo) & 0.66 & 0.32 & \\
\hline $12 \mathrm{Mo}$ & $23.2 \pm 0.4$ & $22.2 \pm 0.6$ & 0.16 \\
\hline P value (baseline vs.12 mo) & 0.12 & 0.80 & \\
\hline $18 \mathrm{Mo}$ & $22.4 \pm 0.7$ & $23.6 \pm 0.9$ & 0.21 \\
\hline P value (baseline vs.18 mo) & 0.56 & 0.71 & \\
\hline \multicolumn{4}{|l|}{$\lg G(g / L)$} \\
\hline Baseline & $11.1 \pm 0.3$ & $10.7 \pm 0.3$ & 0.42 \\
\hline 3 Days & $11.3 \pm 0.3$ & $11.0 \pm 0.2$ & 0.34 \\
\hline P value (baseline vs.3d) & 0.41 & 0.39 & \\
\hline $1 \mathrm{Mo}$ & $11.5 \pm 0.4$ & $10.9 \pm 0.4$ & 0.13 \\
\hline$P$ value (baseline vs.1 mo) & 0.10 & 0.63 & \\
\hline
\end{tabular}

Table 3 Quantitative measures of blood index (Continued)

\begin{tabular}{llll}
\hline 4 Mo & $10.9 \pm 0.4$ & $10.3 \pm 0.4$ & 0.48 \\
P value (baseline vs.6 mo) & 0.74 & 0.36 & \\
12 Mo & $11.8 \pm 0.3$ & $11.3 \pm 0.4$ & 0.31 \\
P value (baseline vs.12 mo) & 0.09 & 0.13 & \\
18 Mo & $11.6 \pm 0.5$ & $11.1 \pm 0.5$ & 0.18 \\
P value (baseline vs.18 mo) & 0.11 & 0.64 & \\
IgM(g/L) & & & \\
Baseline & $1.10 \pm 0.10$ & $1.07 \pm 0.08$ & 0.80 \\
3 Days & $1.15 \pm 0.09$ & $1.05 \pm 0.07$ & 0.38 \\
P value (baseline vs.3d) & 0.24 & 0.67 & \\
1 Mo & $1.24 \pm 0.11$ & $1.23 \pm 0.10$ & 0.69 \\
P value (baseline vs.1 mo) & 0.28 & 0.15 & \\
4 Mo & $1.17 \pm 0.10$ & $1.05 \pm 0.08$ & 0.35 \\
P value (baseline vs.6 mo) & 0.50 & 0.69 & \\
12 Mo & $1.14 \pm 0.06$ & $1.15 \pm 0.05$ & 0.55 \\
P value (baseline vs.12 mo) & 0.66 & 0.19 & \\
18 Mo & $1.35 \pm 0.11$ & $1.31 \pm 0.10$ & 0.59 \\
P value (baseline vs.18 mo) & 0.39 & 0.24 &
\end{tabular}

\section{CEA(ng/ml)}

$\begin{array}{llll}\text { Baseline } & 2.21 \pm 0.14 & 2.63 \pm 0.17 & 0.08 \\ 3 \text { Days } & 2.47 \pm 0.15 & 2.55 \pm 0.17 & 0.71 \\ \text { P value (baseline vs.3 d) } & 0.03 & 0.48 & \\ 1 \text { Mo } & 2.33 \pm 0.14 & 2.76 \pm 0.19 & 0.11 \\ \text { P value (baseline vs.1 mo) } & 0.33 & 0.40 & \\ 4 \text { Mo } & 2.37 \pm 0.15 & 2.42 \pm 0.15 & 0.91 \\ \text { P value (baseline vs.6 mo) } & 0.25 & 0.21 & \\ 12 \text { Mo } & 2.33 \pm 0.18 & 2.44 \pm 0.16 & 0.62 \\ \text { P value (baseline vs.12 mo) } & 0.58 & 0.34 & \\ 18 \text { Mo } & 2.22 \pm 0.16 & 2.55 \pm 0.24 & 0.16 \\ \text { P value (baseline vs.18 mo) } & 0.21 & 0.34 & \end{array}$

\section{$\mathrm{CRP}$ (mg/L)}

$\begin{array}{llll}\text { Baseline } & 5.72 \pm 0.53 & 6.47 \pm 0.49 & 0.27 \\ 3 \text { Days } & 4.99 \pm 0.51 & 5.47 \pm 0.48 & 0.31 \\ \text { P value (baseline vs.3 d) } & 0.12 & 0.06 & \\ 1 \text { Mo } & 3.70 \pm 0.40 & 3.54 \pm 0.31 & 0.80 \\ \text { P value (baseline vs.1 mo) } & <0.0001 & <0.0001 & \\ 4 \text { Mo } & 3.20 \pm 0.33 & 3.34 \pm 0.30 & 0.54 \\ \text { P value (baseline vs.6 mo) } & <0.0001 & <0.0001 & \\ 12 \text { Mo } & 3.04 \pm 0.19 & 3.00 \pm 0.29 & 0.46 \\ \text { P value (baseline vs.12 mo) } & <0.0001 & <0.0001 & \\ 18 \text { Mo } & 3.56 \pm 0.43 & 3.42 \pm 0.38 & 0.76 \\ \text { P value (baseline vs.18 mo) } & <0.0001 & <0.0001 & \end{array}$


Table 3 Quantitative measures of blood index (Continued)

\begin{tabular}{|c|c|c|c|}
\hline \multicolumn{4}{|l|}{$\mathrm{ALT}(\mathrm{U} / \mathrm{L})$} \\
\hline Baseline & $30.5 \pm 1.8$ & $32.8 \pm 2.1$ & 0.41 \\
\hline 3 Days & $31.6 \pm 2.1$ & $32.9 \pm 2.7$ & 0.85 \\
\hline P value (baseline vs.3 d) & 0.42 & 0.98 & \\
\hline $1 \mathrm{Mo}$ & $30.3 \pm 1.8$ & $28.3 \pm 1.3$ & 0.68 \\
\hline P value (baseline vs.1 mo) & 0.96 & 0.47 & \\
\hline $4 \mathrm{Mo}$ & $29.3 \pm 1.1$ & $29.9 \pm 1.2$ & 0.72 \\
\hline P value (baseline vs.6 mo) & 0.87 & 0.59 & \\
\hline $12 \mathrm{Mo}$ & $27.2 \pm 1.1$ & $27.4 \pm 1.1$ & 0.94 \\
\hline P value (baseline vs.12 mo) & 0.36 & 0.31 & \\
\hline $18 \mathrm{Mo}$ & $29.2 \pm 1.4$ & $28.1 \pm 1.2$ & 0.61 \\
\hline P value (baseline vs.18 mo) & 0.83 & 0.43 & \\
\hline \multicolumn{4}{|l|}{ TBIL $(\mu \mathrm{mol} / \mathrm{L})$} \\
\hline Baseline & $16.7 \pm 1.5$ & $15.1 \pm 1.3$ & 0.63 \\
\hline 3 Days & $11.6 \pm 0.6$ & $11.5 \pm 0.5$ & 0.89 \\
\hline P value (baseline vs.3 d) & 0.0003 & 0.006 & \\
\hline $1 \mathrm{Mo}$ & $15.1 \pm 0.7$ & $13.8 \pm 0.6$ & 0.32 \\
\hline P value (baseline vs.1 mo) & 0.32 & 0.31 & \\
\hline $4 \mathrm{Mo}$ & $14.5 \pm 1.0$ & $16.0 \pm 1.1$ & 0.27 \\
\hline P value (baseline vs.6 mo) & 0.22 & 0.60 & \\
\hline $12 \mathrm{Mo}$ & $14.2 \pm 0.7$ & $15.6 \pm 0.5$ & 0.12 \\
\hline P value (baseline vs.12 mo) & 0.14 & 0.76 & \\
\hline $18 \mathrm{Mo}$ & $14.6 \pm 0.7$ & $13.4 \pm 0.6$ & 0.36 \\
\hline P value (baseline vs.18 mo) & 0.31 & 0.28 & \\
\hline \multicolumn{4}{|l|}{ BUN(mmol/L) } \\
\hline Baseline & $5.30 \pm 0.18$ & $5.63 \pm 0.22$ & 0.28 \\
\hline 3 Days & $4.91 \pm 0.19$ & $5.19 \pm 0.17$ & 0.12 \\
\hline P value (baseline vs.3 d) & 0.06 & 0.07 & \\
\hline $1 \mathrm{Mo}$ & $5.10 \pm 0.18$ & $5.26 \pm 0.23$ & 0.96 \\
\hline P value (baseline vs.1 mo) & 0.36 & 0.22 & \\
\hline $4 \mathrm{Mo}$ & $5.29 \pm 0.17$ & $5.21 \pm 0.18$ & 0.47 \\
\hline P value (baseline vs.6 mo) & 0.95 & 0.13 & \\
\hline $12 \mathrm{Mo}$ & $5.42 \pm 0.18$ & $5.46 \pm 0.20$ & 0.89 \\
\hline$P$ value (baseline vs.12 mo) & 0.68 & 0.57 & \\
\hline $18 \mathrm{Mo}$ & $5.22 \pm 0.23$ & $5.33 \pm 0.32$ & 0.85 \\
\hline P value (baseline vs.18 mo) & 0.46 & 0.29 & \\
\hline \multicolumn{4}{|l|}{ CRE $(\mu \mathrm{mol} / \mathrm{L})$} \\
\hline Baseline & $84.5 \pm 2.3$ & $81.8 \pm 2.2$ & 0.39 \\
\hline 3 Days & $81.2 \pm 2.0$ & $77.9 \pm 2.1$ & 0.26 \\
\hline P value (baseline vs.3 d) & 0.14 & 0.14 & \\
\hline $1 \mathrm{Mo}$ & $78.5 \pm 2.4$ & $76.3 \pm 2.0$ & 0.46 \\
\hline P value (baseline vs.1 mo) & 0.06 & 0.07 & \\
\hline $4 \mathrm{Mo}$ & $83.5 \pm 2.2$ & $78.2 \pm 2.0$ & 0.07 \\
\hline P value (baseline vs.6 mo) & 0.71 & 0.17 & \\
\hline
\end{tabular}

Table 3 Quantitative measures of blood index (Continued)

\begin{tabular}{llll}
\hline 12 Mo & $84.0 \pm 2.0$ & $80.4 \pm 1.8$ & 0.19 \\
$P$ value (baseline vs.12 mo) & 0.84 & 0.63 & \\
18 Mo & $82.6 \pm 2.1$ & $79.7 \pm 2.7$ & 0.48 \\
P value (baseline vs.18 mo) & 0.56 & 0.61 & \\
\hline
\end{tabular}

Plus-minus values are means \pm SE. $P$ values of between-group comparisons were determined by Student's t-test or nonparametric Mann-Whitney $U$ tests. $P$ values of within-group comparisons were determined by ANOVA with $95 \%$ Cls. ALT alanine aminotransferase, ANOVA analysis of variance, BUN blood urea nitrogen, $C E A$ carcino-embryonic antigen, $C l$ confidence interval, $C R P C$ reactive protein, TBIL total bilirubin

had second generation drug eluting stents inserted in primary PCI (Table 1). After five days, they were assigned evenly and randomly to the WJMSC or placebo groups (Fig. 1). The baseline characteristics and clinical interventions of reperfusion and medication were wellmatched in the two groups (Table 1). During the 18month follow-up period in the placebo group, one patient withdrew for missed follow-up due to a move, one patient died 10 days after discharge and one patient could not complete the follow-up at 12 months due to a diagnosis of leukemia. In the WJMSC group, one subject could not complete the four month follow-up due to a diagnosis of advanced stomach cancer (Fig. 1). Therefore, the findings from 58 patients in the WJMSC group and 57 patients in the placebo group were analyzed statistically in this trial as shown in Fig. 1.

\section{Adverse events (AEs)}

The major adverse cardiac events (MACEs) and other clinical AEs encountered are summarized in Table 2. No major peri-procedural complications occurred in either group. As mentioned above, one subject in the placebo group suffered sudden cardiac death 10 days after discharge. In the WJMSC group, one subject was rehospitalized due to heart failure induced by a respiratory infection two weeks post-treatment, and one subject required re-vascularization for in-stent restenosis in the infarct-related coronary artery four months posttreatment. No new arrhythmias were recorded on the Holter monitor study during the 18-month follow-up. The groups did not differ in occurrences of MACEs, including death, recurrences of AMIs and re-hospitalization due to heart failure, during the course of treatment and the 18-month follow-up period.

In the WJMSC group, one patient was diagnosed with advanced stomach cancer three months post-treatment. However, no evidence of ectopic tissue formation and no increase in the levels of tumor-associated antigens were observed in the remaining subjects during the follow-up. WJMSC infusion induced neither acute nor persistent immune or biochemical abnormalities, as shown in Table 3. 


\section{Myocardial viability}

No difference in the intensity of F-18-FDG-PET signals was observed between the WJMSC and placebo group at baseline. However, there was a significantly greater increase in the tracer uptake within the infarcted area from baseline to four months post-treatment in the WJMSC group compared with the placebo group. As shown in Fig. 2a, the absolute increase in the F-18-FDGPET signal intensity was markedly higher in the WJMSC group $(6.9 \pm 0.6 \%, 95 \%$ CI $5.7-8.2)$ than in the placebo group $(3.3 \pm 0.7 \%, 95 \%$ CI $1.8-4.7),(\mathrm{P}<0.0001)$.

\section{Myocardial perfusion}

The 99mTc-SPECT imaging analysis of myocardial perfusion showed a similar total infarcted area at baseline in both groups (Fig. 2b). An increase in myocardial perfusion was observed in both groups four months posttreatment compared with baseline (Fig. 2b). However, as shown in Fig. 2b, there was a significantly higher absolute $99 \mathrm{mTc}$-SPECT signal intensity in the WJMSC group (7.1 $\pm 0.8 \%, 95 \%$ CI 5.4-8.8) compared with the placebo group ( $3.9 \pm 0.6,95 \% \mathrm{CI} 2.8-5.0),(\mathrm{P}=0.002)$.

\section{Left ventricular function}

As shown in Fig. 3 and Table 4, patients treated with WJMSCs experienced a $7.8 \pm 0.9$ (6.0 to approximately 9.7) LVEF increase over baseline at 18 months compared with the $2.8 \pm 1.2$ (0.4 to approximately 5.1) increase in the placebo group ( $\mathrm{p}=0.001)$. Concomitantly, the absolute decreases in LV end-systolic volumes and LV end-diastolic volumes at 18 months in the WJMSC group were significantly greater than those in the placebo group $(\mathrm{P}=0.0004, \mathrm{P}=0.004$, respectively).

\section{Discussion}

In this randomized, double-blind, controlled trial, we reported the safety and efficacy of intracoronary allogeneic WJMSC transplantation in patients with STelevation AMI. A reduction in myocardial infarct size and improved heart function were confirmed by the myocardial viability and perfusion measurements, as well as the global LVEF. Importantly, WJMSC transplantation prevented post-infarct LV adverse remodeling, as evidenced by the changes in the LVEDV and LVESV at 18 months.
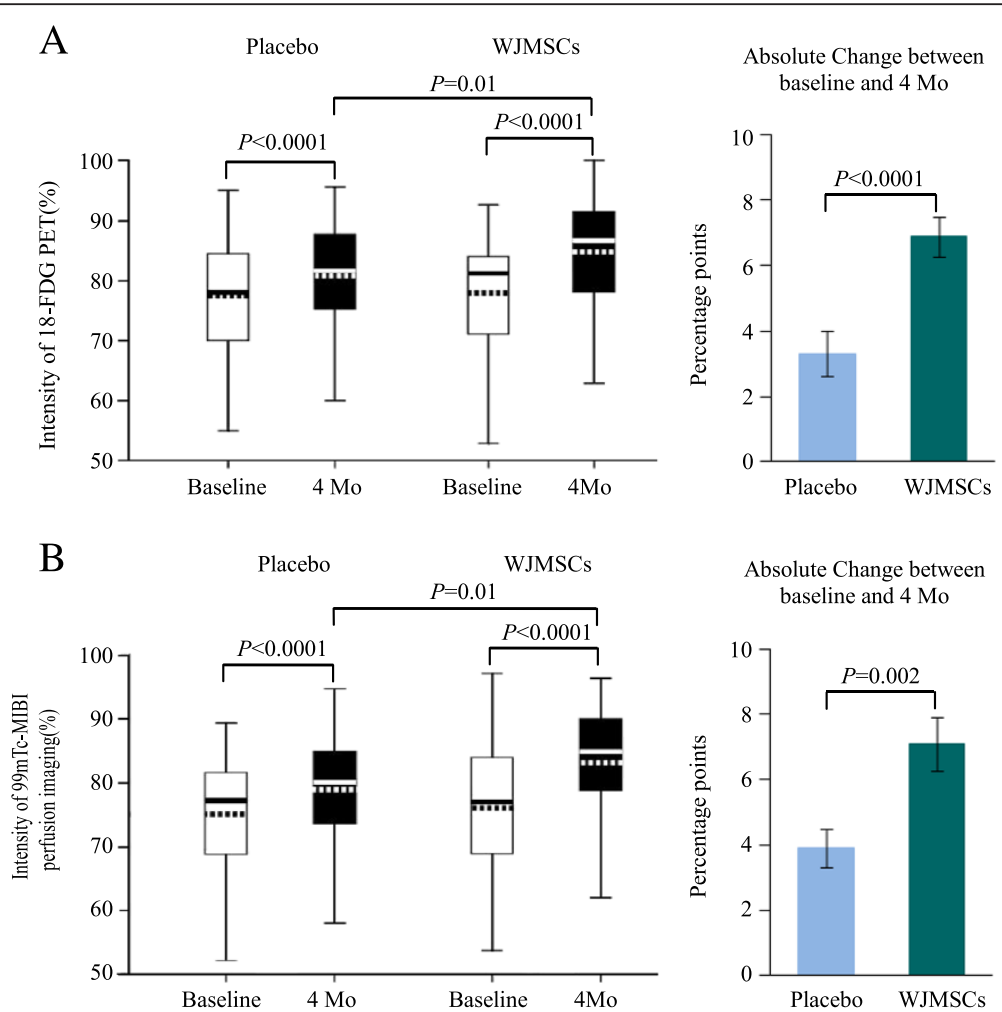

Fig. 2 a Mean F-18-FDG-PET signal intensity in the infarct vessel area measured before and four months after transplantation. Comparison of mean F-18-FDG-PET signal intensity changes and absolute changes between the WJMSC group and the placebo group using analysis of covariance (ANCOVA). T-bars show the standard errors. b Mean signal intensity of 99mTc-MIBI perfusion imaging in the infarcted vessel area measured before and four months after transplantation. Comparison of mean 99mTc-MIBI signal intensity changes and absolute changes between the WJMSC group and the placebo group by ANCOVA. T-bars show the standard errors WJMSC Wharton's jelly-derived mesenchymal stem cells 


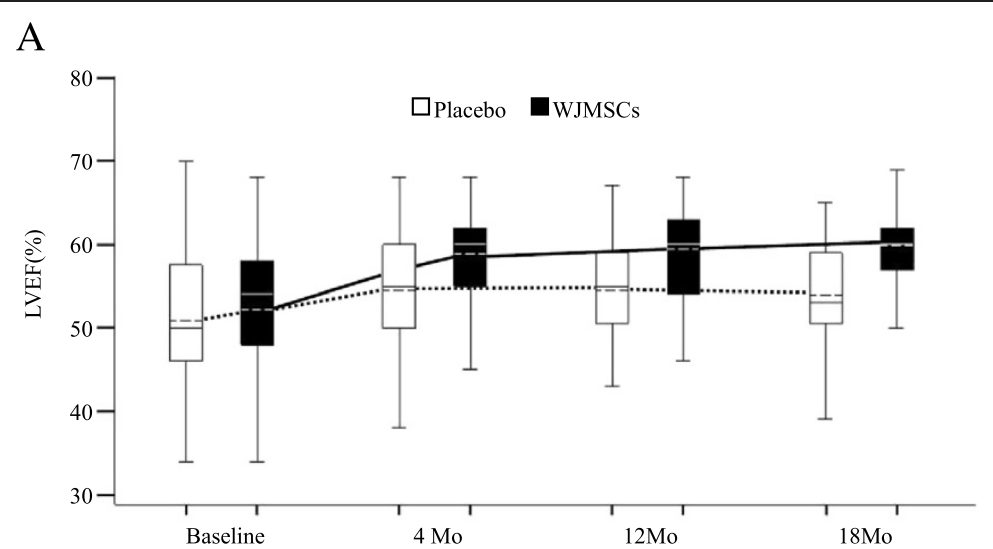

\section{B}
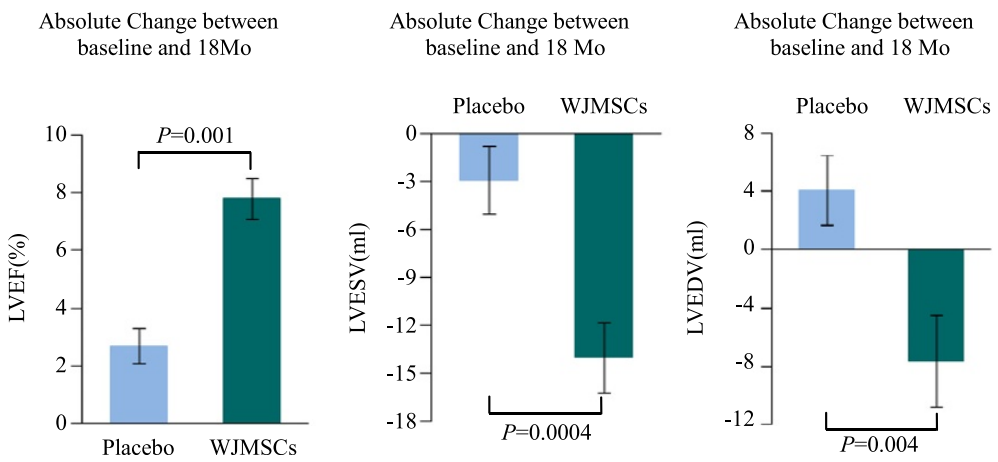

Fig. 3 a Comparison of changes in the global left ventricular ejection fraction (LVEF) between the WJMSC group and the placebo group measured by two dimensional-echocardiograph before transplantation and at the 4-, 12- and 18-month follow-ups. b. The absolute changes in LVEF, LVESV and LVEDV between the WJMSC group and the placebo group at 18 months measured by ECG and analyzed by ANCOVA. T-bars show the standard errors ANCOVA analysis of covariance, ECG echocardiogram, LVEDV left ventricular end-diastolic volume, LVESV left ventricular end-systolic volume, WJMSC Wharton's jelly-derived mesenchymal stem cells

After a heart attack, an optimal window of opportunity exists during which stem cell-based therapies can exert a therapeutic effect [7]. Thus, stem cells that meet clinical grade parameters in terms of purity, potency, identity, and dose must be readily available. However, the desired dose is difficult to obtain with autologous MSCs, because they cannot be expanded and processed in less than 14 days $[12,13]$, thus reducing their usefulness due to missing the optimal time window for cell treatment after AMI. This study provides an exploratory roadmap for the introduction of a promising and urgently needed cellular therapy in clinical practice, based on the concurrent microenvironmental conditions and stage of the disease. It promises an off-the-shelf product for AMI patients, backed by a solid procedure and a validated infrastructure that can be administered to eligible patients during the most favorable time window.

Notably, no signs of an immune response triggered by the allogeneic WJMSC transplantation, ectopic tissue formation or increased levels of tumor-associated antigens were observed in this study. One patient in the WJMSC group was diagnosed with advanced stomach cancer at the three-month follow-up; however, the tumor-associated antigen findings and pathological examination results indicated that the tumor was not likely to have derived from the WJMSCs. In addition, the intracoronary injection of WJMSCs, unlike BMSCs which could be associated with acute complications such as coronary occlusion during transfer surgery [12], did not impair the TIMI flow grade or trigger an increase in troponin concentrations, demonstrating that no microvascular obstruction or coronary artery occlusion had occurred during or after the treatment (Table 2). Taken together, the current results support the conclusion that WJMSC transplantation is relatively safe at least up to 18 months.

Based on previous studies and meta analyses [7-11], there were slight but significant improvements (from 2.5 to $3.66 \%$ of LVEF) in LV function and a significant decrease in the infarct size $-4.03 \%$ of patients with AMI after autologous bone marrow stem cell therapy. Cardiac 
Table 4 Quantitative measures of left ventricular function

\begin{tabular}{|c|c|c|c|}
\hline \multirow[t]{2}{*}{ Variable } & Placebo $(95 \%$ Cl) & WJMSCs(95 \% Cl) & \multirow[t]{2}{*}{$P$ value } \\
\hline & (number = 55) & \multirow{2}{*}{\multicolumn{2}{|c|}{ (number = 57) }} \\
\hline \multicolumn{2}{|l|}{ Global LVEF (\%) } & & \\
\hline \multicolumn{4}{|l|}{ Baseline } \\
\hline Mean & $51.0 \pm 1.1(48.8 \sim 53.3)$ & $52.1 \pm 1.0(50.2 \sim 54.1)$ & 0.46 \\
\hline \multicolumn{4}{|l|}{4 Months } \\
\hline Mean & $54.2 \pm 0.9(52.5 \sim 56.1)$ & $58.5 \pm 0.7(57.1 \sim 60.0)$ & 0.0003 \\
\hline Difference & $3.2 \pm 0.9(1.4 \sim 5.0)$ & $6.4 \pm 0.7(4.9 \sim 7.9)$ & 0.008 \\
\hline P value (baseline vs.6 months) & 0.0009 & $<0.0001$ & \\
\hline \multicolumn{4}{|l|}{12 Months } \\
\hline Mean & $54.5 \pm 0.8(52.8 \sim 56.2)$ & $58.8 \pm 0.8(57.3 \sim 60.4)$ & 0.0002 \\
\hline Difference & $3.5 \pm 0.8(1.9 \sim 5.0)$ & $6.7 \pm 0.8(5.1 \sim 8.3)$ & 0.004 \\
\hline$P$ value (baseline vs.12 months) & $<0.0001$ & $<0.0001$ & \\
\hline \multicolumn{4}{|l|}{18 Months } \\
\hline Mean & $54.0 \pm 0.8(52.3 \sim 55.7)$ & $60.0 \pm 0.5(58.9 \sim 61.0)$ & $<0.0001$ \\
\hline Difference & $2.8 \pm 1.2(0.4 \sim 5.1)$ & $7.8 \pm 0.9(6.0 \sim 9.7)$ & 0.001 \\
\hline P value (baseline vs.18 months) & 0.05 & $<0.0001$ & \\
\hline \multicolumn{4}{|l|}{ LVFS (\%) } \\
\hline \multicolumn{4}{|l|}{ Baseline } \\
\hline Mean & $26.6 \pm 0.7(25.2 \sim 27.9)$ & $27.3 \pm 0.6(26.0 \sim 28.6)$ & 0.42 \\
\hline \multicolumn{4}{|l|}{4 Months } \\
\hline Mean & $29.7 \pm 0.7(28.3 \sim 31.0)$ & $32.6 \pm 0.6(31.4 \sim 33.9)$ & 0.002 \\
\hline Difference & $3.1 \pm 0.6(1.9 \sim 4.3)$ & $5.3 \pm 0.6(4.0 \sim 6.6)$ & 0.01 \\
\hline P value (baseline vs.6 months) & $<0.0001$ & $<0.0001$ & \\
\hline \multicolumn{4}{|l|}{12 Months } \\
\hline Mean & $29.8 \pm 0.6(28.5 \sim 31.0)$ & $32.7 \pm 0.5(31.7 \sim 33.8)$ & 0.0005 \\
\hline Difference & $3.2 \pm 0.5(2.1 \sim 4.3)$ & $5.4 \pm 0.5(4.3 \sim 6.5)$ & 0.005 \\
\hline$P$ value (baseline vs.12 months) & $<0.0001$ & $<0.0001$ & \\
\hline \multicolumn{4}{|l|}{18 Months } \\
\hline Mean & $28.6 \pm 0.6(20.8 \sim 35.6)$ & $33.6 \pm 0.5(27.3 \sim 39.1)$ & $<0.0001$ \\
\hline Difference & $2.1 \pm 0.8(-8.9 \sim 13.5)$ & $6.2 \pm 0.7(-4.1 \sim 16.4)$ & $<0.0001$ \\
\hline P value (baseline vs.18 months) & 0.01 & $<0.0001$ & \\
\hline \multicolumn{4}{|l|}{ WMSI (17-segment model) } \\
\hline \multicolumn{4}{|l|}{ Baseline } \\
\hline Mean & $1.28 \pm 0.03(1.23 \sim 1.34)$ & $1.29 \pm 0.03(1.24 \sim 1.34)$ & 0.76 \\
\hline \multicolumn{4}{|l|}{4 Months } \\
\hline Mean & $1.16 \pm 0.02(1.11 \sim 1.21)$ & $1.10 \pm 0.01(1.07 \sim 1.12)$ & 0.02 \\
\hline Difference & $-0.12 \pm 0.02(-0.16 \sim-0.09)$ & $-0.19 \pm 0.02(-0.23 \sim-0.16)$ & 0.003 \\
\hline P value (baseline vs.6 months) & $<0.0001$ & $<0.0001$ & \\
\hline \multicolumn{4}{|l|}{12 Months } \\
\hline Mean & $1.15 \pm 0.02(1.11 \sim 1.20)$ & $1.09 \pm 0.02(1.06 \sim 1.12)$ & 0.02 \\
\hline Difference & $-0.13 \pm 0.02(-0.17 \sim-0.10)$ & $-0.20 \pm 0.02(-0.23 \sim-0.17)$ & 0.003 \\
\hline P value (baseline vs.12 months) & $<0.0001$ & $<0.0001$ & \\
\hline
\end{tabular}

18 Months 
Table 4 Quantitative measures of left ventricular function (Continued)

\begin{tabular}{|c|c|c|c|}
\hline Mean & $1.16 \pm 0.9(1.02 \sim 1.40)$ & $1.07 \pm 0.01(1.00 \sim 1.17)$ & $<0.0001$ \\
\hline Difference & $-0.13 \pm 0.02(-0.42 \sim 0.08)$ & $-0.22 \pm 0.02(-0.57 \sim 0.04)$ & 0.01 \\
\hline P value (baseline vs.18 months) & $<0.0001$ & $<0.0001$ & \\
\hline \multicolumn{4}{|l|}{ End-systolic volume (ml) } \\
\hline \multicolumn{4}{|l|}{ Baseline } \\
\hline Mean & $66.0 \pm 3.1(59.8 \sim 72.2)$ & $63.1 \pm 2.8(57.5 \sim 68.7)$ & 0.38 \\
\hline \multicolumn{4}{|l|}{4 Months } \\
\hline Mean & $63.4 \pm 2.8(57.7 \sim 69.0)$ & $55.9 \pm 2.1(51.7 \sim 60.2)$ & 0.07 \\
\hline Difference & $-2.6 \pm 1.8(-6.4 \sim 1.1)$ & $-7.2 \pm 1.9(-10.9 \sim-3.5)$ & 0.09 \\
\hline P value (baseline vs.6 months) & 0.16 & 0.0003 & \\
\hline \multicolumn{4}{|l|}{12 Months } \\
\hline Mean & $62.5 \pm 2.8(56.9 \sim 68.0)$ & $55.1 \pm 2.1(50.9 \sim 59.4)$ & 0.06 \\
\hline Difference & $-3.5 \pm 1.9(-7.3 \sim 0.2)$ & $-8.0 \pm 2.0(-12.1 \sim-3.9)$ & 0.12 \\
\hline P value (baseline vs.12 months) & 0.11 & 0.0002 & \\
\hline \multicolumn{4}{|l|}{18 Months } \\
\hline Mean & $62.8 \pm 0.9(59.1 \sim 66.6)$ & $49.2 \pm 1.0(47.1 \sim 51.2)$ & $<0.0001$ \\
\hline Difference & $-2.9 \pm 2.1(-7.1 \sim 1.3)$ & $-14.0 \pm 2.2(-18.4 \sim-9.5)$ & 0.0004 \\
\hline P value (baseline vs.18 months) & 0.2 & $<0.0001$ & \\
\hline \multicolumn{4}{|l|}{ End-diastolic volume (ml) } \\
\hline \multicolumn{4}{|l|}{ Baseline } \\
\hline Mean & $132.2 \pm 3.9(124.3 \sim 140.0)$ & $130.3 \pm 3.8(122.7 \sim 137.9)$ & 0.49 \\
\hline \multicolumn{4}{|l|}{4 Months } \\
\hline Mean & $136.0 \pm 4.0(127.9 \sim 144.0)$ & $133.2 \pm 3.2(126.8 \sim 139.5)$ & 0.72 \\
\hline Difference & $3.8 \pm 2.3(-0.7 \sim 8.4)$ & $2.9 \pm 2.6(-2.3 \sim 8.0)$ & 0.78 \\
\hline P value (baseline vs.6 months) & 0.20 & 0.07 & \\
\hline \multicolumn{4}{|l|}{12 Months } \\
\hline Mean & $134.5 \pm 3.9(126.6 \sim 142.4)$ & $131.9 \pm 3.1(125.8 \sim 138.1)$ & 0.60 \\
\hline Difference & $2.4 \pm 2.5(-2.6 \sim 7.3)$ & $1.6 \pm 2.8(-4.0 \sim 7.2)$ & 0.84 \\
\hline P value (baseline vs.12 months) & 0.45 & 0.22 & \\
\hline \multicolumn{4}{|l|}{18 Months } \\
\hline Mean & $136.4 \pm 3.1(130.1 \sim 142.7)$ & $122.7 \pm 1.6(119.5 \sim 125.8)$ & 0.0001 \\
\hline Difference & $4.1 \pm 2.4(-0.8 \sim 9.0)$ & $-7.6 \pm 3.13(-13.9 \sim-1.4)$ & 0.004 \\
\hline P value (baseline vs.18 months) & 0.1 & $<0.0001$ & \\
\hline
\end{tabular}

LVEF, LVESV and LVEDV by two dimensional-ECG from baseline to 18 months post-treatment between the WJMSC group and placebo-control group were analyzed by ANCOVA. Treatment effects are expressed as differences in least-squares means (ANCOVA model) with $95 \% \mathrm{Cl}$ ANCOVA analysis of covariance, $\mathrm{Cl}$ confidence interval, ECG echocardiogram, LVEDV left ventricular end-diastolic volume, LVESF left ventricular end-systolic volume, WJMSC Wharton's jelly-derived mesenchymal stem cells

regeneration is defined as regrowth of lost or destroyed cardiomyocytes. Here, we verified that the transplanted WJMSCs significantly increased myocardial viability within the infarcted area as measured by F-18-FDG-PET, which is considered sensitive and specific for assessing viable myocardium [32, 33]. Concomitantly, the myocardial perfusion in the infarcted area and the LVEF were increased significantly after WJMSC transplantation. Notably, even though the infarct size $(-6.9 \%)$ was not reduced greatly after the transfer of the WJMSCs, the gradual improvements in LVEDV and LVESV over 18 months in the WJMSC group demonstrated that these cells could prevent adverse LV remodeling effectively. Previous studies have shown that the transfer of BMMCs had no significant impact on LVEDV, suggesting that BMMCs may have a limited effect on LV remodeling after AMI $[7,8]$. Taken together, our findings indicate that combined optimal reperfusion therapy (stent implantation) and 
intracoronary administration of WJMSCs during the optimal time frame can decrease the infarct size, enhance the recovery of global and regional $\mathrm{LV}$ function and prevent LV remodeling after AMI.

Our study was not designed to assess the underlying mechanisms of WJMSC treatment that promote functional recovery after AMI. Nevertheless, evidence supports the idea that WJMSCs constitute a unique cell family with a high degree of stemness and unique transcriptional profiles [34, 35]. We previously reported higher expression levels of mesoderm and specialized cardiac progenitor cell genes, including Brachyury (T), mesoderm posterior 1 , Flk-1, Nkx2.5 and Isl-1, which represent key transcription factors of cardiac development [18]. These key characteristics of WJMSCs indicate their strong potential to differentiate into cardiomyocytes, endothelia, and to form neovascular networks, to integrate into ischemic cardiac tissues, all of which result in the improvement of heart function [18-22]. Moreover, WJMSCs can secrete large amounts of anti-apoptotic, angiogenic factors and growth factors, exerting paracrine effects for the regeneration of myocardium and coronary vessels [36].

Tumorigenesis is a major concern in the clinical application of human ESCs and induced pluripotent stem cells (iPS). Studies have demonstrated that normal karyotypes were observed in WJMSCs harvested from primary, early and late passages, and teratomas were not induced after WJMSC injection into severe combined immune deficient (SCID) mice [37]. No ectopic tissues or increased levels of tumor-associated antigens were observed after WJMSC transplantation in this trial. Furthermore, WJMSCs can regulate immunity by modulating the behavior of natural killer (NK) cells and T-cell populations to evade immune responses and are welltolerated in allogeneic transplantation [24, 26].

In this study, a relatively lower dose of WJMSCs was administered for intracoronary infusion because in our previous clinical trial one patient suffered from a serious complication of coronary artery occlusion during the BMSC injection procedure [12]. However, the wide safety margin found in the present study indicated that higher doses of WJMSCs would be safe for clinical trials in the future.

There is a limitation to this proof-of-concept study. Although we used an especially important tool, PET, for interpreting myocardial viability and predicting improvements in cardiac function, contrast-enhanced magnetic resonance imaging (CE-MRI) was not available in all centers to assess cardiac function. This may be a drawback compared to recently related publications.

\section{Conclusions}

We demonstrated for the first time that intracoronary delivery of prepared clinical-grade WJMSCs, a true stem cell population with highly expressing early cardiac transcription factors, was safe in treating patients with an AMI attack and could significantly improve myocardial viability and heart function. Our original study provides an exploratory roadmap to translate the promising cellular therapy into clinical practice according to the urgency for treatment, the concurrent microenvironmental conditions and the stage of disease. Therefore, this study encourages additional clinical trials to determine whether WJMSCs may serve as a novel allogeneic source for cardiac stem cell-based therapies.

\section{Abbreviations}

99mTc-SPECT: 99mTc-sestamibi single-photon emission computed tomography; AEs: adverse events; AMl: acute myocardial infarction; F-18-FDG-PET: F-18fluorodeoxyglucose positron emission computed tomography; LVEF: Left ventricular ejection fraction; WJMSC: Wharton's jelly-derived mesenchymal stem cell.

\section{Competing interests}

The authors declare that they have no competing interests.

\section{Authors' contributions}

LRG contributed to study design, WJMSC protocol preparation approval, patient enrollment, intracoronary transfer of WJMSCs, clinical follow-up of patients, and writing of the report. XH contributed to study design, cell preparation, and writing of the report. YC contributed to patient enrollment, intracoronary transfer of WJMSCs, and clinical follow-up of patients. NKZ contributed to data collection and data analysis. XLY and HLL contributed to patient enrollment, intracoronary transfer of WJMSCs, and clinical follow-up of patients. ZGW, XYY, CY, and HYC contributed to data and statistical analyses. YW, ZMZ, TCL, CLH, BW, ZMW, YDC, GHC, ZYX, JB, DL, PQ, YY, YCD, $\mathrm{LNL}$, and $X C Y$ contributed to patient enrollment, intracoronary transfer of WJMSCs, and clinical follow-up of patients. LHW contributed to Holter recording and analysis. YHS contributed to echocardiography studies. FG contributed to PET and SPECT studies. ML contributed to writing the paper. YY, HTT, and QAD contributed to study design and safety monitoring. All authors read and approved the final manuscript.

\section{Funding statement}

This project was funded by a grant from the National Advanced Technology Development Project of China (863 Project), (2006AA02Z469),

(2011AA020102), (2013AA020101).

\section{Acknowledgments}

We thank Joel S. Karliner, MD of the University of California, San Francisco and John P. Cooke MD PhD, Professor and Chair of the Department of Cardiovascular Sciences, Houston Methodist Research Institute for their valuable comments and suggestions in writing and revising this manuscript. We also thank the nursing and technical staff of the Coronary Care Unit and Catheterization Laboratory of the 11 Cardiology Centers and the Shenzhen Beike Cell Engineering Research Institute.

\section{Author details}

${ }^{1}$ Center of Cardiology, Navy General Hospital, Beijing, China. ${ }^{2}$ Department of Cardiology, the First People's Hospital of Foshan, Guangdong Province, Foshan, China. ${ }^{3}$ Department of Cardiology, General Hospital of Armed Police Forces, Beijing, China. ${ }^{4}$ Shenzhen Beike Cell Engineering Research Institute, Guangdong Province, Shenzhen, China. ${ }^{5}$ Department of Biostatistics, Peking University First Hospital, the Clinical Research Institute of Peking University, Beijing, China. ${ }^{6}$ Department of Cardiology, the General Hospital of Chinese People's Liberation Army, Beijing, China. ${ }^{7}$ Department of Age Cardiology, the General Hospital of Chinese PLA, Beijing, China. ${ }^{8}$ Department of Cardiology, Beijing Huaxin Hospital, Beijing, China. ${ }^{9}$ Department of Cardiology, the Second Affiliated Hospital of Dalian Medical University, Liaoning Province, Dalian, China. ${ }^{10}$ Department of Cardiology, the Central Hospital of Aerospace Corporation, Beijing, China. ${ }^{11}$ Department of Cardiology, General Hospital of Huabei Oilfield, Huabei Province, Renqiu, China. ${ }^{12}$ Department of Cardiology, 
Beijing Chaoyang Hospital of Capital Medical University, Beijing, China. ${ }^{13}$ Department of Cardiology, Central Hospital of National Petroleum Corporation, Huabei Province, Langfang, China. ${ }^{14}$ Department of Nuclear Medicine, Navy General Hospital, Beijing, China. ${ }^{15}$ Department of Ultrasonic Diagnosis, Navy General Hospital, Beijing, China.

Received: 4 March 2015 Accepted: 12 June 2015

Published online: 10 July 2015

\section{References}

1. Yeh RW, Sidney S, Chandra M, Sorel M, Selby JV, Go AS. Population trends in the incidence and outcomes of acute myocardial infarction. N Engl J Med. 2010:362:2155-65.

2. Rosenzweig A. Cardiac regeneration. Science. 2012;338:1549-50.

3. Toma C, Pittenger MF, Cahill KS, Byrne BJ, Kessler PD. Human mesenchymal stem cells differentiate to a cardiomyocyte phenotype in the adult murine heart. Circulation. 2002;105:93-8.

4. Vela DC, Silva GV, Assad JA, Sousa AL, Coulter S, Fernandes MR, et al. Histopathological study of healing after allogenic mesenchymal stem cell delivery in myocardial infarction in dogs. J Histochem Cytochem. 2009;57:167-76.

5. Perin EC, Tian M, Marini 3rd FC, Silva GV, Zheng Y, Baimbridge F, et al. Imaging long-term fate of intramyocardially implanted mesenchymal stem cells in a porcine myocardial infarction model. PLoS One. 2011;6:e22949.

6. Williams AR, Hare JM. Mesenchymal stem cells: biology, pathophysiology, translational findings, and therapeutic implications for cardiac disease. Circ Res. 2011;109:923-40.

7. Schächinger V, Erbs S, Elsässer A, Haberbosch W, Hambrecht R, Hölschermann $\mathrm{H}$, et al. Intracoronary bone marrow-derived progenitor cells in acute myocardial infarction. N Engl J Med. 2006;355:1210-21.

8. Meyer GP, Wollert KC, Lotz J, Steffens J, Lippolt P, Fichtner S, et al. Intracoronary bone marrow cell transfer after myocardial infarction: eighteen months' follow-up data from the randomized, controlled BOOST (BOne marrOw transfer to enhance ST-elevation infarct regeneration) trial. Circulation. 2006:113:1287-94

9. Chen SL, Fang WW, Ye F, Liu YH, Qian J, Shan SJ, et al. Effect on left ventricular function of intracoronary transplantation of autologous bone marrow mesenchymal stem cell in patients with acute myocardial infarction. Am J Cardiol. 2004;94:92-5.

10. Hare JM, Traverse JH, Henry TD, Dib N, Strumpf RK, Schulman SP, et al. A randomized, double-blind, placebo-controlled, dose-escalation study of intravenous adult human mesenchymal stem cells (prochymal) after acute myocardial infarction. J Am Coll Cardiol. 2009;54:2277-86.

11. Jeevanantham V, Butler M, Saad A, Abdel-Latif A, Zuba-Surma EK, Dawn B. Adult bone marrow cell therapy improves survival and induces long-term improvement in cardiac parameters a systematic review and meta-analysis. Circulation. 2012;126:551-68.

12. Gao LR, Pei XT, Ding QA, Chen Y, Zhang NK, Chen HY, et al. A critical challenge: Dosage-related efficacy and acute complication intracoronary injection of autologous bone marrow mesenchymal stem cells in acute myocardial infarction. Int J Cardiol. 2013;168:3191-9.

13. Fan M, Chen W, Liu W, Du GQ, Jiang SL, Tian WC, et al. The effect of age on the efficacy of human mesenchymal stem cell transplantation after a myocardial infarction. Rejuvenation Res. 2010;13:429-38.

14. Wang HS, Hung SC, Peng ST, Huang CC, Wei HM, Guo YJ, et al. Mesenchymal stem cells in the Wharton's jelly of the human umbilical cord. Stem Cells. 2004;22:1330-7.

15. Troyer $\mathrm{DL}$, Weiss ML. Concise review: Wharton's jelly-derived cells are a primitive stromal cell population. Stem Cells. 2008;26:591-9.

16. Karahuseyinoglu S, Cinar O, Kilic E, Kara F, Akay GG, Demiralp DO, et al. Biology of stem cells in human umbilical cord stroma: in situ and in vitro surveys. Stem Cells. 2007;25:319-31.

17. Kim DW, Staples M, Shinozuka K, Pantcheva P, Kang SD, Borlongan CV. Wharton's jelly-derived mesenchymal stem cells: phenotypic characterization and optimizing their therapeutic potential for clinical applications. Int J Mol Sci. 2013;14:11692-712.

18. Gao LR, Zhang NK, Ding QA, Chen HY, Hu X, Jiang S, et al. Common expression of stemness molecular markers and early cardiac transcription factors in Wharton's jelly-derived mesenchymal stem cells and hESCs. Cell Transplant. 2013;22:1883-900.
19. Nartprayut K, U-Pratya Y, Kheolamai P, Manochantr S, Chayosumrit M, Issaragrisil S, et al. Cardiomyocyte differentiation perinatally-derived mesenchymal stem cells. Mol Med Rep. 2013;7:1465-9.

20. Lupu M, Khalil M, Andrei E, lordache F, Pfannkuche K, Neef K, et al. Integration properties of Wharton's jelly-derived novel mesenchymal stem cells into ventricular slices of murine hearts. Cell Physiol Biochem. 2011;28:63-76.

21. Zhang W, Liu XC, Yang L, Zhu DL, Zhang YD, Chen Y, et al. Wharton's jelly-derived mesenchymal stem cells promote myocardial regeneration and cardiac repair after miniswine acute myocardial infarction. Coron Artery Dis. 2013;24:549-58.

22. Lopez Y, Lutjemeier B, Seshareddy K, Trevino EM, Hageman KS, Musch TI, et al. Wharton's jelly or bone marrow mesenchymal stromal cells improve cardiac function following myocardial infarction for more than 32 weeks in a rat model: a preliminary report. Curr Stem Cell Res Ther. 2013;8:46-59.

23. Can A, Karahuseyinoglu S. Concise review: human cord stroma with regard to the source of fetus-derived stem cells. Stem Cells. 2007;25:2886-95.

24. Weiss ML, Anderson C, Medicetty S, Seshareddy KB, Weiss RJ, VanderWerff I, et al. Immune properties of human umbilical cord Wharton's jelly-derived cells. Cells Stem Cells. 2008;26:2865-74.

25. Bongso A, Fong CY. The therapeutic potential, challenges and future clinical directions of stem cells from the Wharton's jelly of the human umbilical cord. Stem Cell Rev Rep. 2013;9:226-40.

26. Chen H, Zhang N, Li T, Guo J, Wang Z, Yang M, et al. Human umbilical cord Wharton's jelly stem cells: immune property genes assay and effect of transplantation on the immune cells of heart failure patients. Cell Immunol. 2012;276:83-90.

27. Kushner FG, Hand M, Smith Jr SC, King SB, Anderson JL, Antman EM, et al. 2009 Focused Updates: ACC/AHA Guidelines for the Management of Patients With ST-Elevation Myocardial Infarction (updating the 2004 Guideline and 2007 Focused Update) and ACC/AHA/SCAI Guidelines on Percutaneous Coronary Intervention (updating the 2005 Guideline and 2007 Focused Update): a report of the American College of Cardiology Foundation/American Heart Association Task Force on Practice Guidelines. Circulation. 2009;120:2271-306.

28. Gao LR, Wang ZG, Zhu ZM, Fei YX, He S, Tian HT, et al. Effect of intracoronary transplantation of autologous bone marrow-derived mononuclear cells on outcomes of patients with refractory chronic heart failure secondary to ischemic cardiomyopathy. Am J Cardiol. 2006;98:597-602.

29. Halme DG, Kessler DA. FDA regulation of stem-cell-based therapies. N Engl J Med. 2006;355:1730-5.

30. Gao LR, Zhang NK, Chen Y, Wang ZG, Zhu ZM, Yang Y. A dosing study of Wharton's jelly-derived mesenchymal stem cell for intracoronary infusion in a pig model of acute coronary myocardial infarction. Navy Med J. 2011;3:21-8. Chinese.

31. Cerqueira MD, Weissman NJ, Dilsizian V, Jacobs AK, Kaul S, Laskey WK, et al. American Heart Association Writing Group on Myocardial Segmentation and Registration for Cardiac Imaging. Standardized myocardial segmentation and nomenclature for tomographic imaging of the heart: a statement for healthcare professionals from the Cardiac Imaging Committee of the Council on Clinical Cardiology of the American Heart Association. Circulation. 2002;105:539-42.

32. Liu Q, Zhao S, Yan C, Lu M, Jiang S, Zhang Y, et al. Myocardial viability in chronic ischemic heart disease: comparison of delayed-enhancement magnetic resonance imaging with $99 \mathrm{mTc}$-sestamibi and 18-F-fluorodeoxyglucose single-photon emission computed tomography. Nucl Med Commun. 2009;30:610-6.

33. Mylonas I, Beanlands RS. Radionuclide imaging of viable myocardium: is it under- utilized? Curr Cardiovasc Imaging Rep. 2011:4:251-61.

34. Nekanti U, Rao VB, Bahirvani AG, Jan M, Totey S, Ta M. Long-term expansion and pluripotent marker array analysis of Wharton's jelly-derived mesenchymal stem cells. Stem Cells Dev. 2010;19:117-30.

35. Fong CY, Chak LL, Biswas A, Tan JH, Gauthaman K, Chan WK, et al. Human Wharton's jelly stem cells have unique transcriptome profiles compared to human embryonic stem cells and other mesenchymal stem cells. Stem Cell Rev Rep. 2011;7:1-6.

36. Sobolewski K, Malkowski A, Bankowski E, Jaworski S. Wharton's jelly as a reservoir of peptide growth factors. Placenta. 2005;26:747-52.

37. Fong CY, Richards M, Manasi N, Biswas A, Bongso A. Comparative growth behaviour and characterization of stem cells from human Wharton's jelly. Reprod Biomed Online. 2007;6:708-18. 